\title{
Magnetic-resonance-guided Focused Ultrasound Surgery in the Treatment of Oncology Patients - Fundamentals and Review of Early Clinical Applications
}

\author{
Pedro Valero, ${ }^{1}$ Emilio Gomez-Gonzalez, ${ }^{2}$ Araceli Sabino, ${ }^{3}$ Maria Valero ${ }^{3}$ and Jerónimo Suárez-Ramos ${ }^{4}$ \\ 1. Director of Oncology, Hospital Infanta Luisa, Seville; 2. Director, Group of Interdisciplinary Physics, Department of Applied Physics III, School of Engineering, \\ University of Seville; 3. Researcher and Medical Oncologist; 4. Director of Radiology, Instituto Cartuja
}

\begin{abstract}
Magnetic-resonance-guided focused ultrasound surgery (MRgFUS) is a novel non-invasive surgical procedure in the field of image-guided interventions. Thermal ablation is achieved using focused ultrasound beams controlled by magnetic resonance. Energy delivered to focal (sonication) spots allows for a very precise and controlled increase in temperature, resulting in coagulative necrosis of target tissue. 3D visualisation and temperature monitoring allow for realtime feedback and control. Post-treatment evaluation is performed in the same session. Since there is no dose limit, the procedure can be repeated as needed. An expanding number of applications are currently being developed in oncology. This article reviews the fundamentals and initial clinical data, including palliative treatment of pain in bone metastases, breast cancer, diseases of the central nervous system, prostate cancer, hepatocellular carcinoma and targeted drug delivery.
\end{abstract}

\section{Keywords}

Magnetic resonance, focused ultrasound, non-invasive surgery, image-guided surgery, cancer, tumour ablation, palliative treatment, targeted drug delivery

Disclosure: The Group of Interdisciplinary Physics of the University of Seville and Instituto Cartuja develop collaborative magnetic-resonance-guided focused ultrasound surgery research projects with InSightec Ltd.

Acknowledgements: The authors thank Elena Garcia Laffite, Daniel Ramírez Martínez and Andrés Coca de la Torre for their help. Comments and analysis from Javier Márquez Rivas and Manuel Perales Esteve are also gratefully acknowledged.

Received: 30 June 2010 Accepted: 27 July 2010 Citation: European Oncology, 2010;6(2):76-9 DOI: 10.17925/EOH.2010.06.02.76

Correspondence: Emilio Gomez-Gonzalez, Director, Interdisciplinary Physics Research Group (GFI), Department of Applied Physics III, Engineering School,

University of Seville, Camino de los Descubrimientos s/n, 41092 Seville, Spain. E: egomez@us.es

Support: The publication of this article was funded by InSightec Ltd. The views and opinions expressed are those of the authors and not necessarily those of InSightec Ltd.

Magnetic-resonance-guided focused ultrasound surgery (MRgFUS) is a novel non-invasive therapeutic technique combining an MR imaging device and a focused ultrasound system, also called high-intensity focused ultrasound (HIFU). Concentration of ultrasound beams produces a highly localised (and controlled) increase in temperature (range $60-90^{\circ} \mathrm{C}$ ) at the focal concentration (sonication) spot and thermal ablation of the tissue. ${ }^{1}$ MR allows for precise 3D segmentation of the target tissue and realtime measurement of the temperature at the selected target and surrounding areas. Following injection of a local anaesthetic at the treatment site, the patient lies on a therapeutic table inside the MR system. Acoustic coupling between the transducer and the body and local cooling is achieved by degassed water circulation and gel pads. The treatment takes two or four hours depending on the indication. Recovery time is about one hour after completion of the outpatient procedure. MRgFUS treatment is accomplished using a closed-loop strategy comprising three steps (see Figure 1). The first stage is 3D planning of the procedure, which includes identification of the desired target and definition of position, size and physical features (energy, frequency, duration) of sonication spots. Sonication spots are ellipsoidal, ranging from 1 to $10 \mathrm{~mm}$ in diameter and from 10 to $50 \mathrm{~mm}$ in length. The energy delivered ranges from 1,000 to 2,500 in bursts lasting 15-25 seconds. Sonications overlap to ensure that no tumour is left. This also includes consultation of a previous computer simulation to determine energy levels both at the target and in adjacent areas that need protecting. The second stage is the treatment stage, and involves delivery of the energy. Realtime temperature mapping yields the actual values achieved and provides active feedback during the procedure, allowing changes to be made to the sonication parameters if needed. The third stage of treatment is realtime evaluation of the results and a post-treatment contrast MR study. This allows for precise 3D evaluation of the degree of target destruction achieved. All three steps are performed in the same treatment session. Since there is no dose limit, the procedure can be repeated in the same or different sessions if required, depending on lesion size, type or location.

\section{Available Equipment}

There are two commercially available focused ultrasound systems guided by MR: the ExAblate ${ }^{\circledR}$ MRgFUS system (Insightec Ltd, Haifa, Israel), integrated with a 1.5/3.0T MR scanner (GE, Milwaukee, WI, US), and the Sonalleve ${ }^{\circledR}$ MR-HIFU system (Koninklijke Philips Electronics NV, Eindhoven, The Netherlands). The ExAblate 2000 system became commercially available in 2004, and other models of the family have been launched since then. It has US Food and Drug Administration (FDA) approval for the treatment of uterine fibroids (2004) and CE marks for the treatment of uterine fibroids (2002), palliative treatment of bone metastases (2007) and treatment of adenomyosis (2010). As discussed 
below, other applications, including FDA studies for palliative treatment of bone metastases and breast cancer, are in phase II/III clinical trials. The Sonalleve Philips system received the CE mark for treatment of uterine fibroids in 2009. Published articles in oncology refer to the EXAblate MRgFUS system only.

\section{Current Use in the Treatment of Painful Bone Metastases}

Bone is the third most common organ of metastatic dissemination after the lungs and the liver, and $50-75 \%$ of patients with bone metastases present with severe pain. Palliation of associated symptoms is the primary objective of their treatment. Current options for pain management include both local and systemic therapies. Local options include radiotherapy, surgery and percutaneous ablation by radiofrequency. Systemic treatments comprise hormone therapy, analgesics, chemotherapy and bisphosphonates. ${ }^{2}$

MRgFUS treatment of bone metastases is based on the high acoustic absorption and low thermal conductivity of cortical bone, which allows the use of low energy levels (compared with treatment of soft tissues) to achieve temperatures $>60^{\circ} \mathrm{C}$ at the target locations. This energy produces a coagulative necrosis without damaging the surrounding tissues, which otherwise would receive a sublethal dose. ${ }^{3.4}$

Two mechanisms have been postulated to explain the analgesic effect of MRgFUS: thermal denervation of the periosteum and ablation of the tumoral mass itself. In published studies, immediate pain relief is described, supporting the first hypothesis. Also, a maintained analgesic effect is found, which is explained by both mechanisms. Most treated patients present an improvement on pain scale and a reduced dose of opioids and other analgesics. Possible complications are of a thermal nature, including cutaneous burns and damage to nearby structures that are sensitive to heat, such as nerves or the intestinal wall. None of these side effects has been described in published studies, and realtime imaging control and temperature feedback through thermal mapping make such complications rare. ${ }^{5-7}$

MRgFUS treatment of painful bone metastases has CE mark approval and is currently used in Germany, Italy, Russia and Spain. A case example is shown in Figure 2. There is an ongoing FDA pivotal study to evaluate the effectiveness and safety of the ExAblate system in the palliative treatment of pain from metastatic bone and multiple myeloma tumours in patients who are not suitable candidates for radiotherapy. ${ }^{8}$ This study is in the recruitment phase and is expected to include patients with osteolytic or osteoblastic lesions caused by certain clinical conditions.

\section{Application to Breast Cancer}

Breast cancer constitutes the second cause of cancer-related death in women in developed countries and is a global health issue. ${ }^{9,10}$ Conservative surgery followed by radiotherapy has become the gold standard in the treatment of localised breast cancer. ${ }^{11}$ This procedure has a low mortality rate, but complications can occur, including bleeding (2-10\%), infection (1-20\%), seroma formation (10-80\%) and chronic pain in the incision area (20-30\%). ${ }^{12}$ Technical advances in recent decades have allowed the emergence of minimally and non-invasive techniques such as cryotherapy, interstitial laser ablation, radiofrequency ablation or ultrasound ablation..$^{13-16}$ MRgFUS constitutes a potentially powerful new tool for the treatment of breast cancer.

\section{Figure 1: Closed-loop Strategy for Magnetic-resonance- guided Focused Ultrasound Surgery}

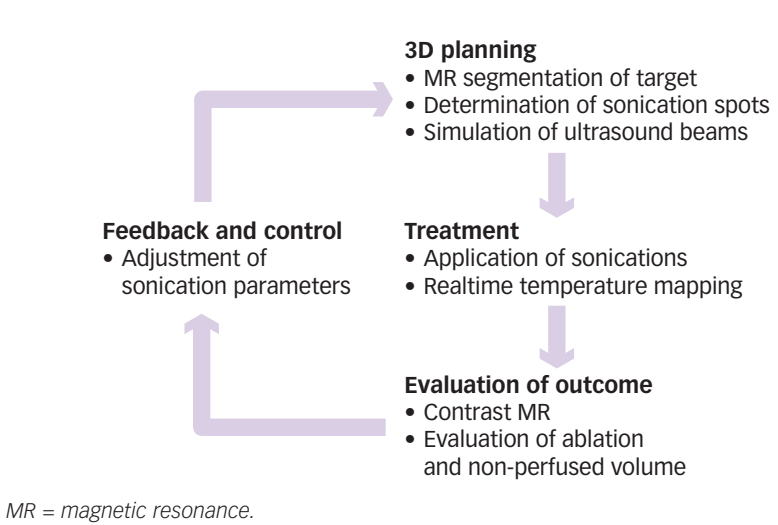

Figure 2: Case Example of Palliative Treatment Using Magnetic-resonance-guided Focused Ultrasound Surgery
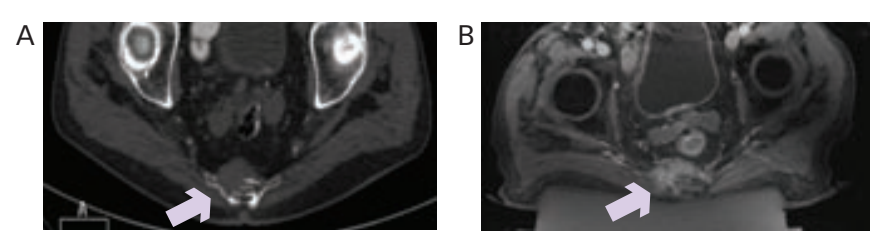

A. Magnetic resonance image (MRI) showing soft-tissue mass within the sacral area with bone destruction. B. Immediate post-treatment MR (T1W contrast-enhanced subtraction image) showing ablated area. The patient is a 72-year-old male with a diagnosis of urothelial carcinoma of the bladder followed by bone metastasis. MR-guided focused ultrasound surgery (MRgFUS) ablation of the coccyx lesion was applied. Before treatment, the patient's pain score was 8 on a on a visual analogue scale (VAS) of 0-10. The same day he reported a very significant symptomatic improvement in pain down to a score of 3 . The next day he reported disappeance of pain (VAS 0). After four-month follow-up, the patient reported no pain and continued improvement in general status.

source: valero et al., 2010.50

The first experience with MRgFUS in the treatment of benign tumours was published by Hynynen and colleagues in 2001. ${ }^{17}$ In the same year, Huber and colleagues published the first case of a malignant breast tumour treated using this technique. ${ }^{18}$ It is important to note that for a non-invasive technique to be considered equivalent to surgical extirpation, it must show necrosis of $100 \%$ of the target tumour. ${ }^{19,20}$ Although there is no clearly established protocol, the combination of MR and biopsy is used as the procedure of choice for detecting residual tumour. To evaluate the safety of margin ablation, Furosawa and colleagues ${ }^{21}$ scheduled a follow-up including MR and ultrasound every three months. Gianfelice ${ }^{22}$ also used MR 10 days and one, three and six months after the treatment as well as multiple biopsies of the affected area within six months. In cases of residual tumour, it is possible to re-treat with MRgFUS and perform a new biopsy one month later.

A number of factors must be considered when selecting candidates for the procedure, ${ }^{23,24}$ including a minimum distance between the tumour and the skin (to avoid skin burns) and between the tumour and the chest wall (to avoid heat accumulation in the ribs and lung). Also, the patient should be able to lie on her chest for several hours during the process. In certain cases, this positioning may become difficult. Although there are no exact recommendations regarding the size of the target lesion, breast tumours $>5 \mathrm{~cm}$ in diameter should not be selected for treatment with MRgFUS because of the increased treatment time and reduced probability of tumour necrosis. ${ }^{25}$ Patients with tumours $\leq 2 \mathrm{~cm}$ in diameter show a $100 \%$ success rate. ${ }^{26}$ Patients with ductal carcinoma in situ (DCIS) should also be excluded from treatment with MRgFUS because MR may under- or overestimate the extent of DCIS, which may 
lead to over- or, even worse, undertreatment. ${ }^{27}$ Furthermore, although the anatomy of the lymphatic drainage of the breast may be altered by hyperthermal coagulative necrosis, published results show that the sentinel node procedure is not affected in $91 \%$ of cases, although the anatomy of the lymphatic drainage of the breast may be altered by hyperthermal coagulative necrosis. ${ }^{28,29}$

\section{Initial Application in the Central Nervous System}

Central nervous system (CNS) tumours include those that originate in the brain and in the spinal cord. They account for $9.2 \%$ of all neoplasms (malignancies) and represent the second leading cause of death from neurological disease after stroke. The incidence of CNS tumours increases in the seventh decade of life. All intracranial tumours, although properly differentiated and relatively benign, are potentially lethal due to their confinement in the skull. Brain tumours do not usually originate distant metastases due to the absence of lymphatic paths, although they may spread to the neuraxis or at the intracerebral level. Currently, the mainstay of their curative treatment is surgery. However in a high proportion of cases surgical options are not possible because of tumour location, relationship with neighbouring vital structures and difficulties with ensuring adequate resection margins. In such cases, other procedures are considered, including chemotherapy, radiation therapy, a combination of both or even proton therapy. Thus, noninvasive treatment of brain tumours represents one of the potential areas of MRgFUS application that is generating the greatest interest. ${ }^{3}$ The safety of intracranial application of MRgFUS has been reported by Hynynen based on a pre-clinical study on primates where there was no need for bone opening thanks to the use of a novel helmet system. ${ }^{31}$ The first non-invasive treatment of three human glioblastoma multiforme cases was recently published by McDannold and colleagues. ${ }^{32}$ The same system was used for non-invasive thalamotomy for treatment of neuropathic pain, as published in 2009 by Martin and colleagues..$^{33}$ The results show the safety and accuracy of non-invasive deep brain lesioning. These data support ongoing research in this field with the aim of developing a truly non-invasive neurosurgical procedure. Other possible applications of MRgFUS in the CNS field arise in functional neurosurgery. Ablation of a path or a beam in the brain may be helpful to treat certain symptoms and diseases. Potential applications include treatment of epilepsy and psychiatric disorders and the therma destruction of the spinothalamic tract for treatment of neuropathic pain. MRgFUS has also been used to treat acute brain infarction, although this application will not be described in this article.

\section{Initial Application in Prostate Cancer}

Prostate carcinoma is a public health problem worldwide, with an increasing incidence due to ageing of the population. It is estimated that in 2015, 450,000 new cases will be diagnosed in the US alone. Current treatments for prostate cancer include surgery, radiotherapy and brachytherapy. The most important complications related to radical prostatectomy include deep vein thrombosis, pulmonary embolism, urinary incontinence and impotence. Radiation therapy has similar complications plus gastrointestinal toxicity. The search for image-guided minimally/non-invasive methods with a low mortality rates is of great interest..$^{35} \mathrm{New}$ therapies include cryotherapy, ${ }^{36}$ photodynamic therapy ${ }^{37}$ and HIFU. The first results of application of HIFU to localised prostate carcinoma were published by Gelet et al. ${ }^{38}$ Pre-clinical MRgFUS trials have been carried out in canine models. The key differences between MR-guided and ultrasound-guided systems is the ability of MR to yield realtime temperature mapping and procedure feedback during the ablation. The possibility of performing treatment in a single session with a low complication rate and without the need to irradiate the patient explain the very high interest in using MRgFUS to treat prostate cancer.

\section{Treatment of Hepatocellular Carcinoma}

As in other malignancies, the gold standard for the treatment of hepatocellular carcinoma is surgical resection. However, about $85 \%$ of patients are not suitable candidates for surgery, and the five-year survival rate is very low. This situation has fostered the development of novel minimally invasive techniques such as microwave and laser ablation, cryotherapy and radiofrequency ablation. The interest in using MRgFUS instead of these techniques is due to its true non-invasive character: since there is no need to insert any type of guide or needle into the hepatic parenchyma, the risk of surgical complications is eliminated. Starting with focused ultrasound, studies have been published on animal models and individual cases. ${ }^{39-41}$ There are still some limitations to be overcome, including the ability to treat lesions behind the ribs and synchronise sonications with respiratory motion. ${ }^{42}$

\section{Application to Targeted Drug Delivery}

The application of MRgFuS to targeted drug delivery is a potentially great innovation and advance in the field of medical oncology since it may help to prevent systemic toxicities and achieve increased intratumoral efficiency in the treatment of solid tumours. One method under development is encapsulated chemotherapy inside heat-sensitive liposomes that remain intact at temperatures around $37^{\circ} \mathrm{C}$. Since malignant tumours are associated with high neovascularisation rates, liposomes would preferably accumulate in the interstitium around the tumour. ${ }^{43}$ Controlled liberation and activation of the drug by MRgFUSinduced hyperthermia would act specifically on the target tumour. ${ }^{44,45}$ Further studies are still needed to investigate suitable drugs and the exact mechanisms of deployment of therapeutic targets. Other future directions of MRgFUS research include the induction of antitumour immune response by activating dendritic cells ${ }^{46}$ and gene transfer by induced hyperthermia. ${ }^{47-49}$

\section{Conclusions}

Non-invasive MRgFUS constitutes a powerful new surgical tool with an increasing variety of applications in oncology, ranging from palliative pain treatment to tumour ablation. Its impact in the management of such health problems as breast, prostate, liver and brain tumours will became clear as data from ongoing trials emerge. The application of this technique to targeted drug delivery has the potential to reduce the side effects and increase the efficiency of oncology treatments.

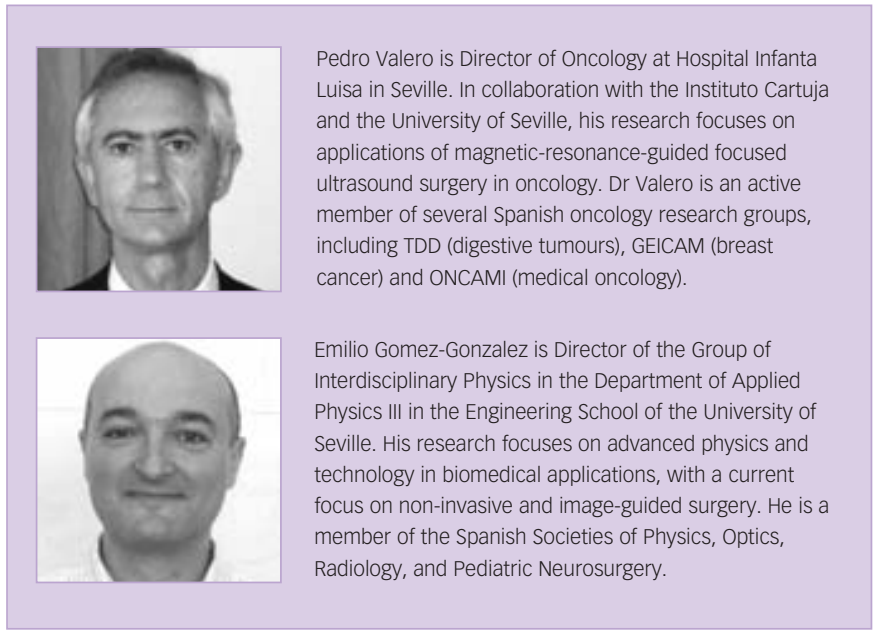


1. Kopelman D, Papa M, Magnetic resonance-guided focused ultrasound surgery for the noninvasive curative ablation of tumors and palliative treatments: a review, Ann Surg Oncol, 2007;14:1540-50.

2. Salvaggi G, Scagliotti GV, Management of bone metastases in cancer: a review, Crit Rev Oncol Hematol, 2005;56:365-78.

3. Smith NB, Temkin JM, Shapiro F, Hynynen K, Thermal effects of focused ultrasound energy on bone tissue, Ultrasound Med Biol, 2001;27:1427-33.

4. Chung AH, Jolesz FA, Hynynen K, Thermal dosimetry of a focused ultrasound beam in vivo by magnetic resonance imaging, Med Phys, 1999;26:2017-26.

5. Catane R, Beck A, Inbar $Y$, et al., MR-guided focused ultrasound surgery (MRgFUS) for the palliation of pain in patients with bone metastases-preliminary clinical experience, Ann Oncol, 2006;18:163-7.

6. Liberman B, Gianfelice D, Inbar Y, et al., Pain palliation in patients with bone metastases using MR-guided focused ultrasound surgery: a multicentre study, Ann surg Oncol, 2009;16(1):140-46.

7. Gianfelice D, Gupta C, Kucharczyk W, et al., Palliative treatment of painful bone metastases with MR imagingguided focused ultrasound, Radiology, 2008;249(1):355-63.

8. ExAblate (Magnetic Resonance-guided Focused Ultrasound Surgery) Treatment of Metastatic Bone Tumors for the Palliation of Pain (NCT00656305), Sponsored by Insightec, last updated 8 April 2010. Available at: ClinicalTrials.gov (accessed June 2010)

9. Globocan 2008, Cancer Fact Sheet. International Agency for Research on Cancer, World Health Organization. Available at: larc.fr (accessed June 2010).

10. Ahmedin J, Thomas A, Murray T, Thun M, Cance statistics 2002, Ca Cancer I Clin, 2002;52(1):23-47.

11. Fisher B, Anderson S, Bryant J, Marglose RG, Twenty year follow up of a randomized trial comparing lumpectomy plus irradiation for the treatment of invasive breast cancer, N Eng/ J Med, 2002;347:1233-41.

12. Veronesi $U$, Cascinelli $N$, Mariani $L$, et al., Twenty year follow up of a randomized trial comparing breastconserving surgery with radical mastectomy for early breast cancer, N Eng J Med, 2002;347:1227-32.

13. Hall-Craggs MA, Interventional MRI of the breast: minimally invasive therapy, Eur Radiol, 2000;10:59-62.

14. Rui J, Tatsutani KN, Dahiya R, Rubinsky B, Effect of thermal variables on human breast cancer in cryosurgery, Breast Cancer Res Treat, 1999;53:185-92.

15. Harms SE, MR-guided minimally invasive procedures, Magn Reson Imaging Clin N Am, 2001;9:381-92.

16. Goldberg SN, Radiofrequency tumor ablation: principles and techniques, Eur J Ultrasound, 2001;13:129-47.

17. Hynynen K, Pomeroy O, Smith DN, et al., MR imagingguided focused ultrasound surgery of fibroadenomas in the breast: a feasibility study, Radiology, 2001;219:176-85

18. Huber PE, Jenne JW, Rastert R, et al., A new noninvasive approach in breast cancer therapy using magnetic resonance imaging-guided focused ultrasound surgery, Cancer Res, 2001;61:8441-9447.
19. Furusawa $\mathrm{H}, \mathrm{Namba}$, Nakahara H, et al., The evolving non-surgical ablation of breast cancer: MR guided focused ultrasound (MRgFUS), Breast Cancer, 2007;14:55-8.

20. Gianfelice D, Khiat A, Amara M, et al., MR imagingguided focused US ablation of breast cancer: histopathologic assessment of effectiveness-initial experience, Radiology, 2003;227:849-55.

21. Furusawa H, Namba K, Thomasen S, et al., Magnetic Resonance-Guided Focused Ultrasound Surgery of Breast Cancer: Reliability and Effectiveness, J Am Coll surg, 2006;203(1):54-63

22. Gianfelice $D$, Khait $A$, Boulanger $Y$, et al., Feasibility of magnetic resonance imaging-guided focused ultrasound surgery as an adjunct to tamoxifen therapy in high-risk surgical patients with breast carcinoma, I Vasc Interv Radiol, 2003;14:1275-1282.

23. Gombos EC, Kacher DF, Furusawa $H$, Namba K, Breast focused ultrasound surgery with magnetic resonance guidance, Top Magn Reson Imaging, 2006;17(3):181-8.

24. Gianfelice $D$, Khiat $A$, Amara M, et al., MR imagingguided focused ultrasound surgery of breast cancer: histopathologic assessment of efficacy - initial experience, Radiology, 2003;227(3):850-55.

25. Gianfelice D, Khiat A, Amara M, et al., MR imagingguided focused ultrasound surgery of breast cancer: correlation of dynamic contrast-enhanced MRI with histopathological findings, Breast Cancer Res Treat, 2003:82:93-101.

26. Fornage $B D$, Sneige $N$, Ross $M I$, et al., Small $(<$ or $=2-\mathrm{cm})$ breast cancer treated with US-guided radiofrequency ablation: feasibility study, Radiology, 2004;231:215-524.

27. Schmitz AC, Gianfelice D, Daniel BL, et al., Image-guided focused ultrasound ablation of breast cancer: current status, challenges and future directions, Eur Radiol, 2008;18(7):1431-41.

28. Zippel DB, Papa MZ, The use of MR imaging guided focused ultrasound in breast cancer patients; a preliminary phase one study and review, Breast Cancer, 2005; $12: 32-8$

29. Vargas HI, Dooley WC, Gardner RA, et al., success of sentinel lymph node mapping after breast cancer ablation with focused microwave phased array thermotherapy, Am J Surg, 2003;186:330-32.

30. Ram Z, Cohen ZR, Harnof S, et al., Magnetic resonance imaging-guided high-intensity focused ultrasound for brain therapy, Neurosurgery, 2006;59(5):949-56.

31. Hynynen K, McDannold N, Clement G, Jolesz FA, PreClinical testing of a phased array ultrasound system for MRI-guided noninvasive surgery of the brain - a primate study, Eur J Radiol, 2006;59(2):149-56.

32. McDannold N, Clement GT, Black P, et al., Transcranial magnetic resonance imaging-guided focused ultrasound surgery of brain tumors: initial findings in three patients, Neurosurgery, 2010;66(2):323-32.

33. Martin E, Jeanmonod D, Morel A, et al., High-intensity focused ultrasound for noninvasive functional neurosurgery, Ann Neurol, 2009;66:858-61.
34. Bradley WG, MR-guided focused ultrasound: a potentially disruptive technology, J Am Coll Radiol, 2009;6(7):510-13.

35. Tempany C, Straus S, Nobuhiko H, Haker S, MR-guided prostate interventions, J Magn Reson Imaging, 2008;27(2): 356-67.

36. Bahn DK, Lee F, Cryosurgical Ablation therapy for prostate cancer, Arch Ital Urol Androl, 2000;72:302-4.

37. Weersink RA, Bogaards A, Gertner M, et al., Techniques for delivery and monitoring TOOKAD(WST09)-mediated photodynamic therapy of the prostate: clinical experience and practicalities, J Photochem Photobiol, 2005;79:211-22.

38. Gelet A, Chapelon JY, Bouvier R, et al., Treatment of prostate cancer with transrectal focused ultrasound: Early clinical experience, Eur Urol, 1996;29:174-83.

39. Cheng SQ, Zhou XD, Tang ZY, et al., High-intensity focused ultrasound in the treatment of experimental liver tumour, J Cancer Res Clin Oncol, 1997;123(4):219-23.

40. Kopelman D, Inbar Y, Hanannel A, et al., Magnetic Resonance-guided Focused Ultrasound: Ablation of liver tissue in a porcine model, Eur J Radiol, 2006;59:157-62.

41. Jolesz FA, Hynynen K, MCDannold N, et al., Noninvasive thermal ablation of hepatocelular carcinoma using magnetic resonance imaging-guided focused ultrasound, Gastroenterology, 2004;127:s242-s247.

42. Okada A, Murakami T, Mikami K, et al., A case of hepatocellular carcinoma treated by MR-guided focused ultrasound ablation with respiratory gating, Magn Reson Med Sci, 2006;5(3):167-71.

43. Allen TM, Liposomes: opportunities in drug delivery, Drugs, 1997;4:8-14.

44. Kong G, Braun RD, Dewhirst MW, Characterization of the effect of hyperthermia on nanoparticle extravasation fron tumor vasculature, Cancer Res, 2001;61:3021-2.

45. Dittmar KM, Xie J, Hunter F, et al., Pulsed high-intensity focused ultrasound enhances systemic administration of naked DNA in squamous cell carcinoma: initial experience, Radiology, 2005;235:541-6.

46. Hu H, Yang XY, Liu Y, et al., Investigation of HIFUinduced antitumor activity in a murine tumour model, I Trans Med, 2007:5:34.

47. Rome C, Couillard F, Moonen CTW, Gene expression and gene therapy imaging, Eur Radiol, 2007;17:305-19.

48. Moonen CTW, Spatio-temporal control of gene expression and cancer treatment using magnetic resonance imaging-guided focused ultrasound, Clin Cancer Res, 2007;13:3482-9.

49. Plathow C, Lohr F, Divkovic G, et al., Focal gene induction in the liver of rats by heat-inducible promoter using focused ultrosound hyperthermia, Inv Radiology, 2005;40:729-7835.

50. Valero P, Suarez J, Gomez-Gonzalez E, et al., Palliative treatment of pain in bone metastases by magnetic resonance guided focused ultrasound surgery (MRgFUS): Initial 4-case experience in Spain, World Conference on Interventional Oncology (WCIO), Philadelphia, US, 2010 